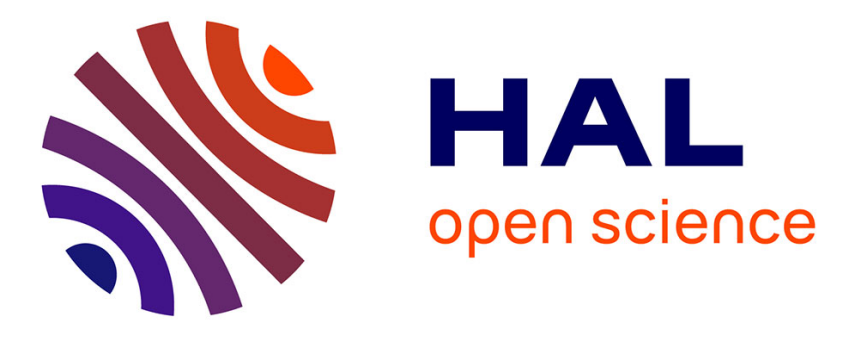

\title{
Numerical simulations of gas-particle flows with combustion
}

Julien Nussbaum, Philippe Helluy, Jean-Marc Hérard, Alain Carrière

\section{To cite this version:}

Julien Nussbaum, Philippe Helluy, Jean-Marc Hérard, Alain Carrière. Numerical simulations of gasparticle flows with combustion. Flow, Turbulence and Combustion, 2006, 76 (4), pp.403-417. hal00139626

\section{HAL Id: hal-00139626 https://hal.science/hal-00139626}

Submitted on 3 Apr 2007

HAL is a multi-disciplinary open access archive for the deposit and dissemination of scientific research documents, whether they are published or not. The documents may come from teaching and research institutions in France or abroad, or from public or private research centers.
L'archive ouverte pluridisciplinaire HAL, est destinée au dépôt et à la diffusion de documents scientifiques de niveau recherche, publiés ou non, émanant des établissements d'enseignement et de recherche français ou étrangers, des laboratoires publics ou privés. 


\title{
Numerical simulations of gas-particle flows with combustion
}

\author{
Julien NUSSBAUM \\ French-german Research Institut of Saint-Louis \\ 5, rue du Général Cassagnou \\ BP 34, 68301 SAINT LOUIS CEDEX \\ Philippe HELLUY \\ Laboratoire ANAM/MNC, ISITV \\ BP 56, 83162 LA VALETTE CEDEX \\ Jean-Marc HÉRARD \\ LATP - UMR CNRS 6632 \\ 39 rue Joliot Curie. 13453 MARSEILLE CEDEX 13 \\ Dpt MFTT, EDF - Recherche et Développement \\ 6 quai Watier. 78401 CHATOU CEDEX
}

Alain CARRIÈRE

French-german Research Institut of Saint-Louis,

5 rue du Général Cassagnou,

BP 34, 68301 SAINT LOUIS CEDEX.

november 2005

Abstract. This work is devoted to the numerical modelling of a reactive gasparticle flow that arises in internal ballistic. The model, proposed by Gough [2], takes into account complex physical phenomena such as mass transfer, drag force or intra granular stress. A non-conservative finite volume approach adapted from [11] is applied in order to simulate the model. After an academic validation test case of the scheme, the combustion propagation ignited by a cylindrical perforated primer is then simulated and compared with experiments.

Keywords: finite volume, non-conservative scheme, two-phase flow, solid propellant, ignition, internal ballistic

\section{Internal ballistic problem}

The two-phase flows in guns are very difficult to model. Many inter phase interaction and complex phenomena occur while the powder burns and the bullet moves in the gun tube.

In order to simplify the physical geometry of a gun, we consider a cylindrical combustion chamber linked to a tube of the same constant cross section. At the initial time, the mixture of gas-powder grains is contained in the combustion chamber, limited by the breech at one end, and by the shot base at the other end. The initial geometry is illustrated on Figure 1.

(C) 2006 Kluwer Academic Publishers. Printed in the Netherlands. 
Propellant is ignited by a hot gas stream from an igniter. The igniter is a cylinder filled with black powder, perforated by several holes. Hot combustion gases escape through the holes. The addition of energy increases the propellant grain surface temperature. The combustion occurs when the ignition temperature is reached.

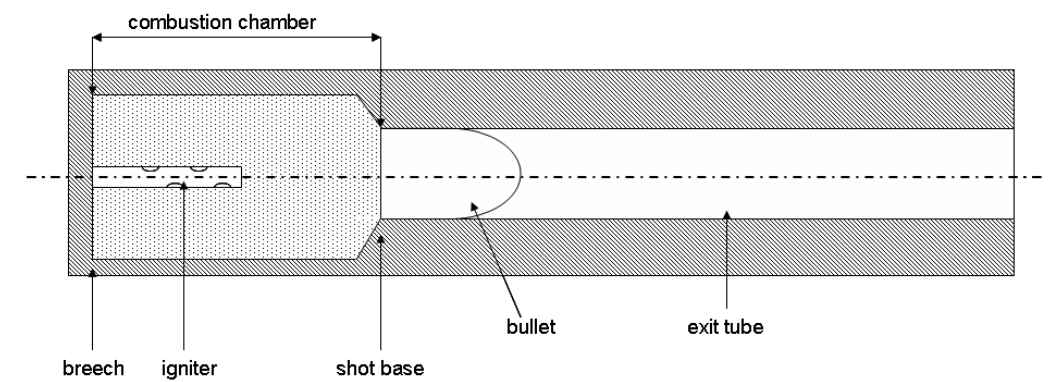

Figure 1. Initial geometry in a gun

After ignition, the solid propellant burns and gases are produced. We assume that the combustion products are similar to the initial gas species. The pressure increases in the combustion chamber, while the front flame propagates in the powder bed according to the following mechanism: ignition of some grains produces a hot gas stream (as igniter does) that locally increases the temperature and pressure. The gases propagate in the domain, and by local heat transfer from the gas phase to the solid phase, the other grains are ignited. The bullet begins to move when the pressure at the shot base is greater than the start pressure, and goes into the tube until the muzzle. Internal ballistic studies stop when the bullet exits from the tube.

Experimental studies exist but are limited: pressure measurements are only possible at the breech or at the shot base and the only available velocity is the bullet's velocity at the muzzle. The temperature and pressure gradients, the high velocities of the flow and the particles volume fractions are still difficult to measure.

\section{The mathematical model}

One can find many two-phase flow models in the scientific literature, focusing on different approaches, with different advantages and drawbacks. We refer for example to the book of Gidaspow [1] for a survey of such models. We will concentrate here on the Gough's model [2] that is very popular in the ballistic community. Our work is devoted to find a numerical method in order to replace a Mac-Cormack integrator 
that is not able to simulate non-classical weapons because of numerical instabilities. For details on the evolution of ballistic models, see [3].

The system is made of the mass and momentum conservation laws for both phases, energy conservation for the gas phase and two convection equations for the enthalpy of the grains and the thickness of burnt powder. The flow is supposed to be one-dimensional in the direction of the symmetry axis of the gun tube with constant section.

In a first stage after ignition, the shot base does not move. In this case, the governing set of equations takes the form

$$
\frac{\partial W}{\partial t}+\frac{\partial F(W)}{\partial x}+C(W) \frac{\partial W}{\partial x}=S(W),
$$

The vector $W=W(x, t) \in \Omega \subset \mathbb{R}^{7}$ is the unknown vector. The time variable is noted $t \in] 0, T$ [ and the space variable $x \in] 0, L[$, where $L$ is the length of the tube. The flux vector $F$ and the sources vector $S$ are functions from $] 0, L[\times] 0, T\left[\right.$ to $\mathbb{R}^{7}$ and $C$ is a function from $] 0, L[\times] 0, T[$ to $\mathbb{R}^{7 \times 7}$. We define

$$
\begin{gathered}
W=\left(\begin{array}{c}
\alpha_{2} \\
\alpha_{1} \rho_{1} \\
\alpha_{1} \rho_{1} u_{1} \\
\alpha_{2} \rho_{2} u_{2} \\
\alpha_{1} E_{1} \\
H_{t s} \\
d
\end{array}\right), \quad F(W)=\left(\begin{array}{c}
\alpha_{2} u_{2} \\
\alpha_{1} \rho_{1} u_{1} \\
\alpha_{1}\left(\rho_{1} u_{1}^{2}+p_{1}\right) \\
\alpha_{2}\left(\rho_{2} u_{2}^{2}+p_{2}\right) \\
\alpha_{1} u_{1}\left(E_{1}+p_{1}\right) \\
0 \\
0
\end{array}\right),\left(\begin{array}{c}
0 \\
0 \\
-p_{1} \partial_{x} \alpha_{1} \\
p_{1} \partial_{x} \alpha_{1} \\
p_{1} \partial_{x}\left(\alpha_{2} u_{2}\right) \\
u_{2} \partial_{x} H_{t s} \\
u_{2} \partial_{x} d
\end{array}\right) \\
C(W) \frac{\partial W}{\partial x}=\left(\begin{array}{c} 
\\
\cos _{2}
\end{array}\right)
\end{gathered}
$$

where $\alpha_{k}$ is the volume fraction, $\rho_{k}$ the density, $u_{k}$ the velocity, $p_{k}$ the pressure and $E_{k}$ the total energy of phase $k$. The index $k=1$ corresponds to the gas phase and $k=2$ corresponds to the solid phase. The solid phase is supposed to be incompressible. The specific enthalpy of the grains is noted $H_{t s}$ and the thickness of the burnt powder of each grain is noted $d$.

REMARK 1. In the sequel, we will give a relation between $p_{2}$ and $p_{1}$ (see formula (8)). It will then be possible to eliminate the pressure $p_{2}$ in the system (1)-(2). Thus, the Gough's system falls into the category of the so-called two-velocity one-pressure models for two-phase flows. 
REMARK 2. In our application, the pressure $p_{1}$ in the solutions of (1)(2) appears to be smooth: the apparition of shock waves would indicate a bad functioning of the gun. Thus, it is not necessary to give a more precise definition of the non-conservative products in (2).

Let us define the source terms by

$$
S(W)=\left(\begin{array}{c}
-\frac{\Gamma_{c}}{\rho_{2}} \\
\Gamma_{c}+\Gamma_{i g n} \\
\Gamma_{c} u_{2}-D+\Gamma_{i g n} u_{i g n} \\
-\Gamma_{c} u_{2}+D \\
\Gamma_{c}\left(Q_{e x}+\frac{p_{1}}{\rho_{2}}+\frac{u_{2}^{2}}{2}\right)-u_{2} D-A_{s} q_{t}+Q_{i g n} \Gamma_{i g n} \\
\kappa q_{t} \\
\dot{r}
\end{array}\right)
$$

In this source term:

- $\Gamma_{c}$ is the mass transfer rate, due to combustion, from the solid phase to the gas phase;

- $\Gamma_{i g n}$ is the mass addition rate from the igniter;

- $u_{i g n}$ is the gas velocity from the igniter;

- $D$ is the interphase drag force;

- $Q_{e x}$ is the exothermic energy released by the solid phase during the combustion;

- $Q_{i g n}$ is the energy released by the igniter;

- $\dot{r}$ is the combustion rate;

- $q_{t}$ is the heat flux per specific surface unit between the two phases;

- $A_{s}=\alpha_{2} \frac{S_{p}}{V_{p}}$ is the specific surface of the solid phase;

- $S_{p}$ and $V_{p}$ are respectively the instantaneous surface and volume of a propellant grain;

- $\kappa$ corresponds to the thermal diffusivity of the solid phase.

REMARK 3. The thermal diffusion and radiation are taken into account only at the local level: in one grain, and between one grain and the surrounding gas. It means that our model will not be able to predict accurately combustion fronts that are driven by thermal diffusion or radiation. The combustion front will propagate because of the convection of hot gases. Pressure waves can also trigger the combustion by a local increase of the temperature. 
In a second stage, when the pressure in the combustion chamber is greater than the resistive pressure of the bullet, the shot base begins to move.

With a moving boundary, the mesh must be adapted to the expansion of the computing domain. We use a rezoning technique that consists in a change of variables in order to obtain a virtual static computation domain. We introduce the change of variable

$$
\xi=\frac{x}{x_{p}},
$$

where $x$ is the real position and $x_{p}$ is the position of the shot base. Thus, $0 \leq \xi \leq 1$, and we obtain a new expression of the system (1)-(2)

$$
\frac{\partial}{\partial t}\left(x_{p} W\right)+\frac{\partial}{\partial \xi}\left(F(W)-v_{p} \xi W\right)+C(W) \frac{\partial W}{\partial \xi}=x_{p} S(W)
$$

In order to compute the position of the shot base $x_{p}$, we apply the fundamental principle of dynamics

$$
m_{p} \frac{d v_{p}}{d t}=A\left(p_{m}-p_{r}\right),
$$

where $v_{p}=\frac{d x_{p}}{d t}$ is the bullet speed, $m_{p}$ its mass, $A$ the tube section, $p_{m}$ the pressure at the shot base and $p_{r}$ the resistive pressure (induced by the bullet/tube friction, and depending on the geometry of the system). The resistive pressure is supposed to be constant.

We consider the following wall boundary conditions:

- at the breech $u_{1}=u_{2}=0$;

- at the shot base $u_{1}=u_{2}=v_{p}$.

At the initial time, the two phases are supposed to occupy homogeneously the initial volume (the combustion chamber).

\section{Constitutive laws}

The definition of $\alpha_{k}$ as volume fraction (also called porosity) for each phase gives

$$
\alpha_{1}+\alpha_{2}=1 .
$$

The perfect gas equation of state is inappropriate in internal ballistic problems. High temperatures and pressures require using real gas equation of state, such as Noble and Abel law that reads

$$
p_{1}\left(\rho_{1}, e_{1}\right)=\frac{(\gamma-1) \rho_{1} e_{1}}{1-\eta \rho_{1}},
$$


where $\gamma$ is the specific heat ratio and $\eta$ is the gas covolume. The internal specific energy $e_{1}$ of the gas is given by

$$
E_{1}=\rho_{1}\left(e_{1}+\frac{u_{1}^{2}}{2}\right) .
$$

$p_{2}$ is defined by

$$
p_{2}\left(\alpha_{1}, \rho_{1}, e_{1}\right)=p_{1}\left(\rho_{1}, e_{1}\right)+R_{p}\left(\alpha_{1}, \rho_{2}\right),
$$

$R_{p}$ is the intra granular stress, given in [4],

$$
R_{p}=\left\{\begin{array}{cl}
0 & \text { if } \alpha_{1}>\alpha_{c} \\
\frac{\rho_{2} c_{p}^{2} \alpha_{c}\left(\alpha_{c}-\alpha_{1}\right)}{\alpha_{1}\left(1-\alpha_{1}\right)} & \text { if } \alpha_{1} \leq \alpha_{c}
\end{array}\right.
$$

The critical porosity $\alpha_{c}$ is usually taken equal to the initial porosity. The velocity $c_{p}$ is a measured sound speed in a particle bed far from compaction. For our configuration, it is sufficient to suppose that it is constant.

From the intra granular stress expression (9), it is possible to compute the speed of propagation of infinitesimal granular disturbances. It is noted $a$ and in the sequel we shall call it the sound speed of the powder. Of course, when the porosity $\alpha_{1}$ is far from 0 , it is much smaller than the real sound speed of the incompressible compacted powder. We have

$$
a^{2}=\frac{R_{p}}{\rho_{2}}-\frac{\alpha_{2} R_{p, \alpha_{1}}}{\rho_{2}} .
$$

In our configuration, the velocity $a$ is given by

$$
a=\left\{\begin{array}{ccc}
0 & \text { if } \alpha_{1}>\alpha_{c} \\
\frac{\alpha_{c}}{\alpha_{1}} c_{p} & \text { if } \quad \alpha_{1} \leq \alpha_{c}
\end{array} .\right.
$$

We observe that the intra granular stress is zero when the porosity (the volume fraction of gas) is sufficiently high. On the other hand, it tends to infinity when the porosity tends to zero. This latter case corresponds to the compaction of the powder. It is clear that the compaction will produce a very high sound speed in the powder. In numerical simulations this can lead to a very constraining CFL condition. Fortunately, in our application, it appears that the combustion of the powder tends to increase the porosity during the computation and that the dominant sound speed is the sound speed of the gas. Hyperbolicity of the system (1)-(2) depends directly on the intra granular stress. A sufficient strength implies hyperbolicity of the system. The eigenvalues of the Gough's model are computed in Appendix B. For more details, 
we refer to [5], [10].

Theoretical expressions have been developed to model the drag force of a single particle in a gas flow, but the more complex case of a particle bed has been modelled only from correlations. We use a limit of Ergun's correlation given in $[6]$

$$
D=f_{r} \frac{\varphi\left(\alpha_{2}\right)}{6} \rho_{2}\left(1-\alpha_{2}\right) \frac{S_{p}}{V_{p}}\left(u_{1}-u_{2}\right)\left|u_{1}-u_{2}\right|,
$$

with $f_{r}$ the resistive factor. The function $\varphi$ depends on the shape of the grains. Here we use

$$
\varphi\left(\alpha_{2}\right)= \begin{cases}0.3 & \alpha_{2}>0.9, \\ 1.75\left(\frac{1-\alpha_{2}}{\alpha_{2}} \frac{\alpha_{c}}{1-\alpha_{c}}\right)^{0.45} & \alpha_{c}<\alpha_{2}<0.9, \\ 1.75 & \alpha_{2}<\alpha_{c} .\end{cases}
$$

At last, we use the combustion law of Vieille [7]

$$
\dot{r}=a_{r} P^{n}+b,
$$

where $a_{r}, b$ and $n$ are experimentally determined constants.

We describe the mass transfer rate by

$$
\begin{aligned}
\Gamma_{c} & =A_{s} \rho_{2} \dot{r} \\
& =\left(1-\alpha_{1}\right) \frac{S_{p}}{V_{p}} \rho_{2} \dot{r},
\end{aligned}
$$

where $S_{p}$ and $V_{p}$ are respectively the instantaneous surface and volume of a grain powder, computed by geometric formulas depending on the shape of the grains. Possible expressions for $S_{p}$ and $V_{p}$ are given below.

The heat flux $q_{t}$ per unit of surface depends on the gas temperature $T$ and particle surface temperature $T_{p s}$ and is defined as follows

$$
q_{t}=h_{t}\left(T-T_{p s}\right),
$$

with $h_{t}$ the total thermal transfer coefficient, sum of convective and radiative coefficients

$$
h_{t}=h_{c}+h_{r} .
$$

The radiative coefficient is computed by

$$
h_{r}=\varepsilon_{p} \sigma\left(T+T_{p s}\right)\left(T^{2}+T_{p s}^{2}\right),
$$

with the hypothesis that the gas emissivity is equal to unity. $\varepsilon_{p}$ corresponds to the particles emissivity and $\sigma$ to the Stephan-Boltzmann constant. 
The convective coefficient is obtained from the Nusselt number deduced from correlations (see [8] for example). It reads

$$
\mathrm{Nu}=6 \frac{h_{c}}{k} \frac{V_{p}}{S_{p}},
$$

where the Eucken approximation [9] for polyatomic gas gives the thermal conductivity of the gas $k$ using the viscosity coefficient $\mu$, the universal gas constant $R$ and the specific heat at constant volume $c_{v}$

$$
k=\frac{15}{4} R \mu\left(\frac{4}{15} \frac{c_{v}}{R}+\frac{3}{5}\right) .
$$

We follow Porterie [4] in order to find another expression of the Nusselt number

$$
\mathrm{Nu}=2+0.4 \operatorname{Re}_{p}^{2 / 3} \operatorname{Pr}^{1 / 3},
$$

where $\operatorname{Pr}$ is the Prandlt number defined by $\operatorname{Pr}=\mu \mathrm{c}_{\mathrm{p}} / \mathrm{k}$ with $c_{p}$ the specific heat of the fluid at constant pressure. From (20), we deduce the expression of the Prandlt number for polyatomic gas

$$
\operatorname{Pr}=\frac{4 \gamma}{9 \gamma-5},
$$

$\gamma$ corresponding to the specific heat ratio.

The expression of the surface temperature $T_{p s}$ is given after the integration of Fourier's law and by supposing a parabolic temperature profile in a spherical grain. See the Appendix in [4] for more details. We compute $T_{p s}$ from

$$
\begin{aligned}
& T_{p s}=T_{p s_{0}}-\frac{3}{2} \frac{h_{t} H_{t s}}{k_{p}^{2}}+ \\
& {\left[\left(T_{p s_{0}}-\frac{3}{2} \frac{h_{t} H_{t s}}{k_{p}^{2}}\right)^{2}+3 \frac{h_{t} H_{t s} T}{k_{p}^{2}}-T_{p s_{0}}^{2}\right]^{1 / 2}}
\end{aligned}
$$

where $k_{p}$ is the thermal conductivity of particles and $T_{p s_{0}}$ is the initial surface temperature.

\section{Numerical method}

We use a version of the Rusanov scheme, a Godunov scheme based on an approximate Riemann solver. In general, Finite Volume Schemes are used for conservation laws. In our case, non-conservative terms induce an adaptation of the scheme. We follow the idea presented in [11] for a two-fluid two-pressure model. 
In order to approximate the solution, consider a space step $h$, a time step $\tau$, the points $\xi_{i}=x_{i} / x_{p}=i h / x_{p}$ and the instants $t^{n}=n \tau$. The computations cells are $\left.C_{i}=\right] \xi_{i-1 / 2}, \xi_{i+1 / 2}[$.

The solution $\widetilde{W}=x_{p} W$ of (3) is approximated in each cell $C_{i}$ and at each time $t^{n}$ by a constant vector

$$
\widetilde{W}_{i}^{n}=x_{p}^{n} W_{i}^{n} \simeq \widetilde{W}\left(\xi, t^{n}\right), \quad \xi \in C_{i} .
$$

The non-conservative finite volume scheme reads

$$
\begin{aligned}
& h\left(\widetilde{W}_{i}^{n+1}-\widetilde{W}_{i}^{n}\right)+\tau\left(\mathcal{F}_{i+1 / 2}^{n}-\mathcal{F}_{i-1 / 2}^{n}\right) \\
& +\tau\left(\mathcal{G}_{i+1 / 2,-}^{n}-\mathcal{G}_{i-1 / 2,+}^{n}\right)=\tau \widetilde{S}_{i}^{n},
\end{aligned}
$$

where $\widetilde{S}_{i}^{n}=x_{p}^{n} S_{i}^{n}$. We define the numerical conservative flux by the classical Rusanov flux

$$
\mathcal{F}_{i+1 / 2}^{n}=\frac{1}{2}\left(\widetilde{F}_{i+1}^{n}+\widetilde{F}_{i}^{n}\right)-\frac{s_{i+1 / 2}^{n}}{2}\left(\widetilde{W}_{i+1}^{n}-\widetilde{W}_{i}^{n}\right),
$$

with

$$
\widetilde{F}_{i}^{n}=F_{i}^{n}-v_{p}^{n} \xi_{i} W_{i}^{n},
$$

and the numerical non-conservative fluxes by

$$
\begin{aligned}
& \mathcal{G}_{i+1 / 2,-}^{n}=C\left(W_{i}^{n}\right) \frac{\widetilde{W}_{i+1}^{n}+\widetilde{W}_{i}^{n}}{2}, \\
& \mathcal{G}_{i-1 / 2,+}^{n}=C\left(W_{i}^{n}\right) \frac{\widetilde{W}_{i}^{n}+\widetilde{W}_{i-1}^{n}}{2},
\end{aligned}
$$

The velocity $s_{i+1 / 2}$ is the maximal wave speed at the interface $i+1 / 2$. It is defined by

$$
\begin{aligned}
& s_{i+1 / 2}^{n}=\max \left(s_{i}^{n}, s_{i+1}^{n}\right), \\
& s_{i}^{n}=\max \left(\left|\left(u_{1}\right)_{i}^{n}\right|+c_{i}^{n},\left|\left(u_{2}\right)_{i}^{n}\right|+a_{i}^{n}\right) .
\end{aligned}
$$

In practice, we only have to consider the wave speed in the gas phase: in our configuration $c_{i}^{n}$ is always greater than the granular wave speed $a_{i}^{n}$ and $\left|u_{2}-u_{1}\right| \ll c$.

In order to satisfy the CFL stability condition we take the time step as

$$
\tau=\delta \frac{h}{\max _{i, n} s_{i}^{n}}
$$

The CFL number $\delta$ has to be $<1$. In practice, we observe a stability of the scheme when this condition is satisfied. It indicates that the source terms are not stiff compared with the convective terms.

Let us notice that the chosen non-conservative numerical flux (28) is arbitrary. Other choices are possible. They will give the same result 
when the solution pressure is smooth. On the other hand, to compute discontinuous pressure solutions, it is important to define precisely the non-conservative products and the associated numerical approximations. For more details on these topics we refer for example to the work of Sainsaulieu [14] and the cited references. Recently, relaxation two-velocity two-pressure models have been designed where the nonconservative products are associated to linearly degenerated waves and are thus naturally defined [11]. It may be an elegant way to circumvent this difficulty.

The Rusanov scheme is known to be very robust but also very dissipative. This robustness is interesting here because a part of the computation occurs in a non-hyperbolic regime. The hyperbolicity of the model is discussed in Appendix B. For more precise computations it is necessary to use a higher order scheme and to modify the model in such a way that it is always hyperbolic.

REMARK 4. Recently, relaxation models for two-velocity two-pressure flows have been designed that are always hyperbolic [11], [16]. It is possible, for some one-pressure models, to provide an approximation by a two-pressure model. In this approach it is necessary to fix a time scale $\tau_{r}$ at which the two pressures equilibrate. When $\tau_{r} \rightarrow 0$ we recover the one-pressure model. It would be interesting to construct such a twopressure model for approximating the Gough's system (but it is not an immediate application of [11] or [16]).

However, numerical experiments have shown that this approach, with $\tau_{r}=0$, does not permit to eliminate the non-hyperbolic instability on fine meshes [12]. To our knowledge, the construction of a general onepressure hyperbolic model for gas-particle flows is still not achieved.

\section{Numerical results}

\subsection{ACADEMiC VALIDATION}

In order to validate our code, we first simulate a flow with constant pressure and constant velocity. It is a numerical test (no physical meaning) where we consider that no interaction occurs between the two phases and we set the intra granular stress $R_{p}=0$.

The initial condition is made of two different constant states (Riemann problem) in a 1 meter length computation domain.

The solution can be computed explicitly (with a source $S(W)=0$ ). The velocity and pressure remain constant, and the other quantities are simply convected at the constant velocity. The proposed numerical 
Table I. Initial conditions, constant velocity, constant pressure.

\begin{tabular}{|c|c|c|c|c|c|c|c|}
\hline & $\alpha_{1}$ & $\rho_{1}\left(\mathrm{~kg} \cdot \mathrm{m}^{-3}\right)$ & $\rho_{2}\left(\mathrm{~kg} \cdot \mathrm{m}^{-3}\right)$ & $u_{1}\left(\mathrm{~m} \cdot \mathrm{s}^{-1}\right)$ & $u_{2}\left(\mathrm{~m} \cdot \mathrm{s}^{-1}\right)$ & $p_{1}(\mathrm{~Pa})$ & $p_{2}(\mathrm{~Pa})$ \\
\hline Left & 0.5 & 0.870 & 1587 & 100 & 100 & $10^{5}$ & $10^{5}$ \\
\hline Right & 0.8 & 0.512 & 1587 & 100 & 100 & $10^{5}$ & $10^{5}$ \\
\hline
\end{tabular}

scheme (26), (28) has the important property that it preserves exactly the constant pressure and velocity fields. Despite its simplicity, this property is not satisfied by all the classical finite volume schemes. The choice of the pressure law (6) is also important (see [13]). This property also appears to be important to compute more complicated configurations.

Figure 2 displays the results obtained with a 1000 cells mesh at a final time $t=3 \mathrm{~ms}$ (the constant velocity and pressure are not represented).

We notice the high diffusion of the density and porosity by the Rusanov scheme on Figure 2. The method is only first order in time and space. It remains interesting for 1D computations, because it is always possible to refine the mesh.

\subsection{Virtual 132 MM GUN}

Secondary, the simulation of a ballistic cycle in a virtual $132 \mathrm{~mm}$ gun (see [15] and Appendix A) is computed. Only the expected bullet velocity at the muzzle, the maximal pressure at the breech and shot base and shot exit time are available. We use a 100 cells mesh, with the empirical values $\gamma=1.27, \eta=1.0838 \cdot 10^{-3}$ and $\alpha_{c}=0.4225$. The powder used is a 7-holes type and the corresponding geometrical functions are

$$
\begin{gathered}
S_{p}=\pi\left(L_{0}-2 d\right)\left[D_{0}-2 d+7\left(d_{0}+2 d\right)\right]+\frac{\pi}{2}\left[\left(D_{0}-2 d\right)^{2}-7\left(d_{0}+2 d\right)^{2}\right], \\
V_{p}=\frac{\pi}{4}\left(L_{0}-2 d\right)\left[\left(D_{0}-2 d\right)^{2}-7\left(d_{0}+2 d\right)^{2}\right],
\end{gathered}
$$

where $L_{0}, D_{0}$ and $d_{0}$ are respectively the length, the external diameter and the perforation diameter before the combustion.

The evolutions of the breech and shot base pressures on time are displayed on Figure 3. Different simulations with other internal ballistic codes have given realistic ranges for the computed values. Table II summarizes the results and shows that our results are in agreement with the expected ones. 

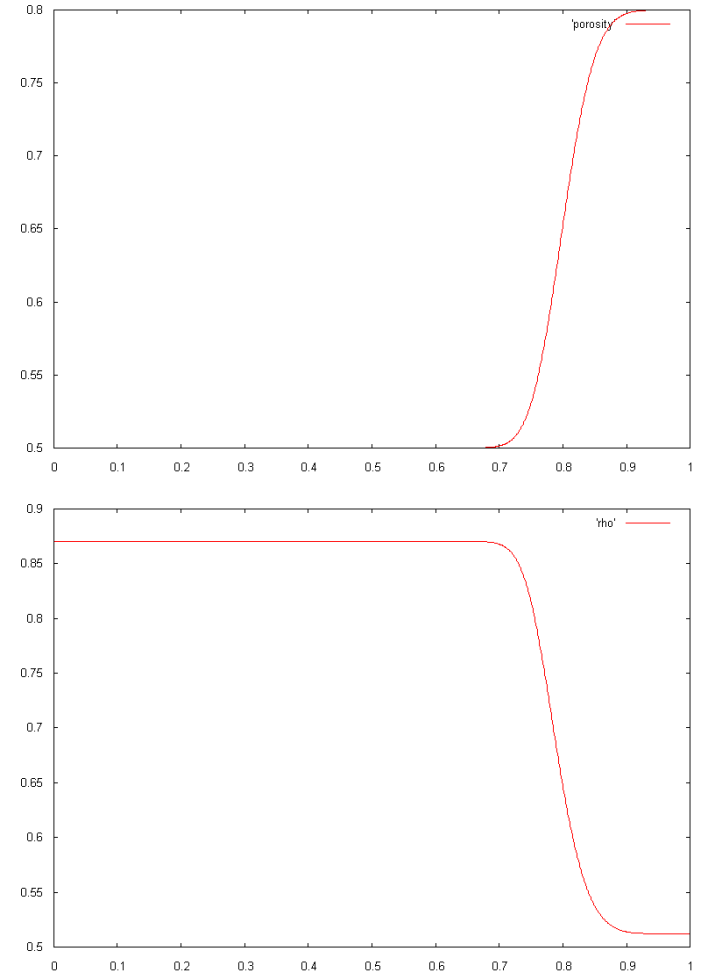

Figure 2. Porosity and density

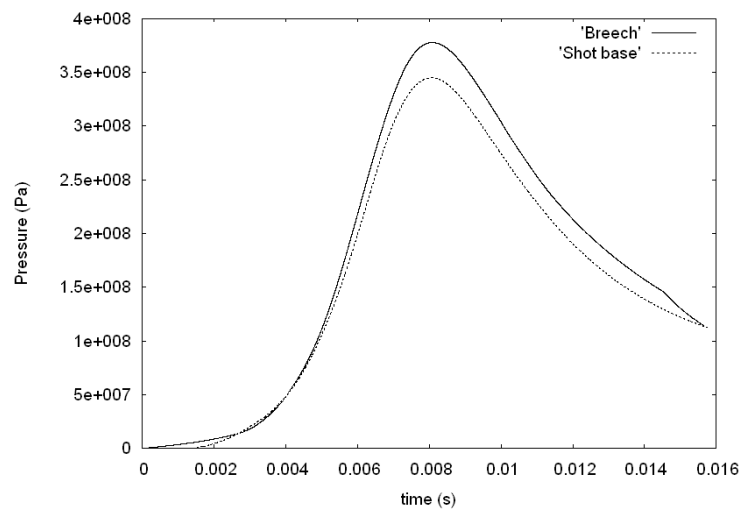

Figure 3. Evolution of pressures 
Table II. Result of simulations

\begin{tabular}{|c|c|c|}
\hline Computed value & Acceptable range & Algorithm result \\
\hline Maximal shot base pressure $(M P a)$ & $325-360$ & 344 \\
\hline Maximal breech pressure $(M P a)$ & $355-400$ & 377 \\
\hline Muzzle velocity $\left(m . s^{-1}\right)$ & $660-705$ & 694 \\
\hline Shot exit time $(m s)$ & $14.66-16.58$ & 15.75 \\
\hline
\end{tabular}

\subsection{REAL 60 MM GUN}

At last, simulations on a $60 \mathrm{~mm}$ gun are compared to experimental measurements given in [17]. We compare the maximal breech pressure and the muzzle velocity coming from the experiments and the simulations, by using three different initial powder masses: $1.17 \mathrm{~kg}, 1.365 \mathrm{~kg}$ and $1.4625 \mathrm{~kg}$. In Table III and IV the data are compared.

Table III. Muzzle velocity datas

\begin{tabular}{|c|c|c|}
\hline Initial powder mass & Experimental result & Simulation result \\
\hline $1.17 \mathrm{~kg}$ & $1000 m \cdot s^{-1}$ & $1001 m \cdot s^{-1}$ \\
\hline $1.365 \mathrm{~kg}$ & $1119 m \cdot s^{-1}$ & $1116 m \cdot s^{-1}$ \\
\hline $1.4625 \mathrm{~kg}$ & $1194 m . s^{-1}$ & $1172 m . s^{-1}$ \\
\hline
\end{tabular}

Table IV. Breech pressure datas

\begin{tabular}{|c|c|c|}
\hline Initial powder mass & Experimental result & Simulation result \\
\hline $1.17 \mathrm{~kg}$ & $367 \mathrm{MPa}$ & $363 M P a$ \\
\hline $1.365 \mathrm{~kg}$ & $534 M P a$ & $521 M P a$ \\
\hline $1.4625 \mathrm{~kg}$ & $665 \mathrm{MPa}$ & $626 \mathrm{MPa}$ \\
\hline
\end{tabular}


We observe a good agreement between the simulations and the experiments even if the precision of the model decreases with the mass of powder.

\section{Conclusion}

In this paper, we have adapted the Gough's model to describe the two-phase flow with combustion in a gun. This is a non-conservative, two-velocity, one-pressure model that involves complicated source terms and lacks of hyperbolicity. We have proposed a numerical method, based on the Rusanov scheme, to simulate this kind of flow. We have been able to obtain satisfactorily results on academic and real configurations.

We have now to improve the model in several directions:

- It is important, for practical configurations, to take into account combustion chambers with variable section;

- It would be interesting to find a relaxation two-velocity two pressure model to approximate the Gough's model. In this way we could avoid the lack of hyperbolicity and then envisage higher order numerical schemes. With an appropriate two-velocity two pressure model we also hope to be able to give a simple definition of the non-conservative products (even if it is not crucial for interior ballistics);

- Finally, a huge work has still to be done on the modelling of the combustion process: chemical model, combustion of grains with special shapes, dependence of combustion front velocity with the convection and the diffusion, etc.

\section{References}

1. D. Gidaspow Multiphase flow and fluidization. Academic Press, 1994.

2. P. S. Gough. Modeling of two-phase flows in guns. AIAA, 66:176-196, 1979.

3. A. Carrière. Sur l'évolution des modèles de Balistique Intérieure de Paul Vieille à nos jours, et leur validation sur diverses armes, des pistolets au canon de Jules Verne. 3èmes Journées Scientifiques Paul Vieille, 19-20 octobre 2000, Paris.

4. B. Porterie. Modélisation de la phase d'allumage d'une charge propulsive en balistique intérieure. PhD Thesis, Aix-Marseille University, 1988. 
5. P. S. Gough and F. J. Zwarts. Modeling of heterogenous two-phase flow. AIAA, 17(1):17-25, 1979.

6. S. Ergun. Fluid flow through packed columns. Chem. Eng. Progress, 48:89-96. 1952.

7. P. Vieille. Étude sur le mode de combustion des substances explosives. 1893.

8. H. Krier, S. Rajan, W. F. Van Tassel. Flame spreading and combustion in packed beds of propellant grains. AIAA, 14(3):301-309, 1976.

9. J. O. Hirschefelder, C. F. Curtiss, R. B. Bird. Molecular theory of gases and liquids. John Wiley $\& 3$ Sons, New-York - London, 1954.

10. L. Combe, J.-M. Hérard. Finite volume algorithm to compute dense compressible gas-solid flows. AIAA, 37(3):337-345, 1999.

11. T. Gallouët, J.-M. Hérard, N. Seguin. Numerical modelling of two-phase flows using the two-fluid two-pressure approach. Mathematical Models and Methods in Applied Science., 14(5):663-700, 2004

12. J.M. Herard, O. Hurisse. A relaxation method to compute two-fluid models, Note EDF HI-81/05/02/A, 2005.

13. T. Gallouët, J.-M. Hérard and N. Seguin. A hybrid scheme to compute contact discontinuities in one-dimensional Euler systems. M2AN Math. Model. Numer. Anal. 36(6):1133-1159 (2003)

14. L. Sainsaulieu. Traveling-wave solutions of convection-diffusion systems in nonconservative form, SIAM J. Math. Anal., 1996, vol. 27-5, pp. 1286-1310.

15. Fluid dynamic aspect of interior ballistics. AGARD fluid dynamic panel, AGARD FDP-AR 172. 1982.

16. R. Saurel and R. Abgrall, A multiphase Godunov method for compressible multifluid and multiphase flows, J. Comp. Phys., 1999, vol. 150, pp. 425-467.

17. A. Kay, Étude de l'influence des charges propulsives à haute densité de chargement, ISL Internal Report, $\mathrm{n}^{\circ}$ 4.4, 2005.

\section{Appendix}

\section{A. Data set for AGARD $132 \mathrm{~mm}$}

We used a 7-holes powder. Igniter's holes are placed at 5 points

$$
\begin{aligned}
& x=0 \mathrm{~mm}, \\
& x=31.75 \mathrm{~mm}, \\
& x=63.5 \mathrm{~mm}, \\
& x=95.24 \mathrm{~mm}, \\
& x=127 \mathrm{~mm} .
\end{aligned}
$$

The computations data are given in Table V. 
Table V. Data set for AGARD $132 \mathrm{~mm}$

\begin{tabular}{|c|c|c|}
\hline $45.359 \mathrm{~d} 0$ & $m_{p}$, projectile weight & $(k g)$ \\
\hline $0.132 \mathrm{~d} 0$ & $A$, calibre (tube section) & $(m)$ \\
\hline 762.d-3 & $x_{p}^{t=0}$, chamber length & $(m)$ \\
\hline $5.08 \mathrm{~d} 0$ & $x_{p, \max }$, tube length & $(m)$ \\
\hline $1 . \mathrm{d} 5$ & $p_{0}$, initial pressure & $(P a)$ \\
\hline 294.d0 & $T_{0}$, initial temperature & $(K)$ \\
\hline 294.d0 & $T_{p s_{0}}$, initial surface temperature & $(K)$ \\
\hline $137.9 \mathrm{~d} 5$ & $p_{r}$, resistive pressure & $(P a)$ \\
\hline $21.3 \mathrm{~d} 0$ & $M$, molecular mass of powder & $(\mathrm{kg} / \mathrm{kmol})$ \\
\hline $1.0838 \mathrm{~d}-3$ & $\eta$, covolume & $\left(m^{3} / k g\right)$ \\
\hline $1.27 \mathrm{~d} 0$ & $\gamma$, specific heat ratio & $(-)$ \\
\hline $9.5255 \mathrm{~d} 0$ & $m_{c}$, powder charge & $(k g)$ \\
\hline 1578.d0 & $\rho_{2}$, density of powder & $\left(k g / m^{3}\right)$ \\
\hline $892.9 \mathrm{~d} 0$ & $Q_{e x}$, explosive heat of powder & $(k c a l / k g)$ \\
\hline $1445.565 \mathrm{~d} 0$ & $c_{v}$, specific heat at constant volume of powder & $\left(m^{2} /\left(s^{2} \cdot K\right)\right)$ \\
\hline 0.d0 & $b$, in the Vieille's law expression & $(\mathrm{m} / \mathrm{s})$ \\
\hline $3.12 \mathrm{~d}-9$ & $a_{r}$, in the Vieille's law expression & $\left(\mathrm{m} / \mathrm{s} / P a^{b}\right)$ \\
\hline $0.9 \mathrm{~d} 0$ & $n$, in the Vieille's law expression & $(\mathrm{SI})$ \\
\hline $11.43 \mathrm{~d}-3$ & $D_{0}$, external diameter of grains & $(m)$ \\
\hline $1.143 \mathrm{~d}-3$ & $d_{0}$, internal diameter of grains & $(m)$ \\
\hline $25.4 \mathrm{~d}-3$ & $L_{0}$, length of a grain & $(m)$ \\
\hline $0.5 \mathrm{~d} 0$ & $f_{r}$, resistance factor of powder & $(-)$ \\
\hline $0.4225 \mathrm{~d} 0$ & $\alpha_{c}$, critical porosity & $(-)$ \\
\hline 254.d0 & $c_{p}$, sound speed in the powder bed & $(m / s)$ \\
\hline $0 . \mathrm{d} 0$ & $\epsilon_{p}$, radiative emission factor & $(-)$ \\
\hline $8.677 \mathrm{~d}-8$ & $\kappa$, thermal conductivity of powder & $\left(m^{2} / s\right)$ \\
\hline $0.2218 \mathrm{~d} 0$ & $\kappa_{p}$, temperature coefficient of powder & $(-)$ \\
\hline $1.5702 \mathrm{~d} 6$ & $Q_{i g n}$, energy released by igniter & $(J / k g)$ \\
\hline 444.d0 & $T_{i g n}$, ignition temperature & $(K)$ \\
\hline $13132 \mathrm{~d} 0$ & $Q_{i g n}$, emission rate & $\left(\mathrm{kg} / \mathrm{m}^{3} / \mathrm{s}\right)$ \\
\hline 10.d-3 & igniter running time & $(s)$ \\
\hline
\end{tabular}

\section{B. Hyperbolicity domain of the model}

We observe first that the hyperbolicity of the model is not affected by the rezoning: it is sufficient to study the eigenvalues of (1)-(2). The last two equations in (1)-(2) are convection equations associated to the eigenvalue $\lambda=u_{2}$. Thus, we concentrate on the first five equations. We rewrite the system in a new set of variables $Y=\left(\alpha_{2}, \rho_{1}, u_{1}, u_{2}, e_{1}\right)$. In 
this way, without the source terms, the system becomes

$$
Y_{t}+B(Y) Y_{x}=0
$$

with

$$
B(Y)=\left[\begin{array}{ccccc}
u_{2} & 0 & 0 & \alpha_{2} & 0 \\
\frac{\rho_{1}}{\alpha_{1}}\left(u_{2}-u_{1}\right) & u_{1} & \rho_{1} & \frac{\rho_{1}}{\alpha_{1}} \alpha_{2} & 0 \\
0 & \frac{p_{1, \rho_{1}}}{\rho_{1}} & u_{1} & 0 & \frac{p_{1, e_{1}}}{\rho_{1}} \\
\frac{R_{p}}{\alpha_{p} \rho_{2}}-\frac{R_{p, \alpha_{1}}}{\rho_{2}} & \frac{p_{1, \rho_{1}}}{\rho_{2}} & 0 & u_{2} & \frac{p_{1, e_{1}}}{\rho_{2}} \\
\frac{p_{1}\left(u_{2}-u_{1}\right)}{\alpha_{1} \rho_{1}} & 0 & \frac{p_{1}}{\rho_{1}} & \frac{p_{1}}{\rho_{1}} \frac{\alpha_{2}}{\alpha_{1}} & u_{1}
\end{array}\right] .
$$

In order to simplify it further, we consider the gas entropy $s\left(\rho_{1}, e_{1}\right)$ satisfying

$$
T_{1} d s=d e_{1}+p_{1} d\left(\frac{1}{\rho_{1}}\right),
$$

where $T_{1}$ is the gas temperature. The entropy also satisfies

$$
\rho_{1} s_{\rho_{1}}=-\frac{p_{1}}{\rho_{1}} s_{e_{1}} .
$$

Multiplying the last equation in (31) by $s_{e_{1}}$ and the second by $s_{\rho_{1}}$ and adding the two we find a new system

$$
\begin{aligned}
& U=\left(\alpha_{2}, \rho_{1}, u_{1}, u_{2}, s\right), \\
& U_{t}+C(U) U_{x}=0, \\
& C(U)=\left[\begin{array}{ccccc}
u_{2} & 0 & 0 & \alpha_{2} & 0 \\
\frac{\rho_{1}}{\alpha_{1}}\left(u_{2}-u_{1}\right) & u_{1} & \rho_{1} & \frac{\rho_{1}}{\alpha_{1}} \alpha_{2} & 0 \\
0 & \frac{p_{1, \rho_{1}}}{\rho_{1}} & u_{1} & 0 & \frac{p_{1, s}}{\rho_{1}} \\
\frac{R_{p}}{\alpha_{2} \rho_{2}}-\frac{R_{p, \alpha_{1}}}{\rho_{2}} & \frac{p_{1, \rho_{1}}}{\rho_{2}} & 0 & u_{2} & \frac{p_{1, s}}{\rho_{2}} \\
0 & 0 & 0 & 0 & u_{1}
\end{array}\right] .
\end{aligned}
$$

The sound speed in the gas is noted $c$. It is given by

$$
c^{2}=\frac{\partial}{\partial \rho_{1}} p_{1}\left(\rho_{1}, s\right)
$$

The sound speed $a$ in the powder bed is given by (11).

In this way, we have

$$
C(U)=\left[\begin{array}{ccccc}
u_{2} & 0 & 0 & \alpha_{2} & 0 \\
\frac{\rho_{1}}{\alpha_{1}}\left(u_{2}-u_{1}\right) & u_{1} & \rho_{1} & \frac{\rho_{1}}{\alpha_{1}} \alpha_{2} & 0 \\
0 & \frac{c^{2}}{\rho_{1}} & u_{1} & 0 & \frac{p_{1, s}}{\rho_{1}} \\
\frac{a^{2}}{\alpha_{2}} & \frac{c^{2}}{\rho_{2}} & 0 & u_{2} & \frac{p_{1, s}}{\rho_{2}} \\
0 & 0 & 0 & 0 & u_{1}
\end{array}\right]
$$


The characteristic polynomial is then

$$
\left(u_{1}-\lambda\right)\left[\left(\left(u_{2}-\lambda\right)^{2}-a^{2}\right)\left(\left(u_{1}-\lambda\right)^{2}-c^{2}\right)-c^{2} \frac{\alpha_{2}}{\alpha_{1}} \frac{\rho_{1}}{\rho_{2}}\left(u_{1}-\lambda\right)^{2}\right]
$$

We define $y=\frac{\lambda-u_{1}}{c}$ that has to be a root of

$$
\begin{aligned}
& Q_{4}(y)=Q_{2}(y) \\
& Q_{4}(y):=\left(y^{2}-1\right)\left(\left(y-\frac{u_{2}-u_{1}}{c}\right)^{2}-\frac{a^{2}}{c^{2}}\right) \\
& Q_{2}(y):=\frac{1-\alpha_{1}}{\alpha_{1}} \frac{\rho_{1}}{\rho_{2}} y^{2}
\end{aligned}
$$

It is easy to check that $Q_{2}$ and $Q_{4}$ have at least two real intersections. For the two other roots, general analytic conditions cannot be obtained but we can indicate sufficient conditions for hyperbolicity (or ellipticity)

1. $a=0, u_{1}=u_{2}$ : the system is hyperbolic.

2. $a=0, u_{1} \neq u_{2},-1 \leq \frac{u_{2}-u_{1}}{c} \leq 1$ : the system is not hyperbolic.

3. $a<c,\left|u_{2}-u_{1}\right| \leq \max (a, c-a)$ : the system is hyperbolic. This sufficient condition is simply obtained by requiring that $Q_{4}(0)>$ $Q_{2}(0)$ when all the roots of $Q_{4}$ are in $[-1,1]$.

Let us observe that in our computations we are generally in the cases 2 or 3. During the ballistic process, the combustion of the powder tends to increase the porosity. Thus, case 3 is observed only at the beginning of the computation, when $\alpha_{1} \simeq \alpha_{c}$ (see (11)). The intra granular stress plays here an important role to stabilize the computation.

Although the rest of the computation occurs in a non-hyperbolic regime we did not observe instabilities. This is certainly due to several dissipative mechanisms:

- the numerical viscosity of the Rusanov scheme is known to be high, compared to other schemes. Typically, if the drag force is set to 0 , instabilities begin to appear for 10000 cells in 1D computations (see [12]);

- the drag force plays an important role to damp the oscillations as demonstrated by Hérard in [12]. The scheme can remain stable up to 500000 cells computations;

- our case corresponds to small velocity differences $u_{2}-u_{1}$ and the size of the imaginary part of the complex eigenvalues tends to 0 with $u_{2}-u_{1}$. 
With finer meshes, or higher order schemes, it will be necessary to improve the stability of the initial model. A simple way to do that is to modify the intra granular stress expression (9) in such a way that $a$ is always $>0$, as in [10] or [5]. 